\title{
RHex: A Biologically Inspired Hexapod Runner*
}

\author{
R. ALTENDORFER \\ Artificial Intelligence Laboratory, University of Michigan, Ann Arbor, MI 48109, USA
}

\author{
N. MOORE \\ Ambulatory Robotics Laboratory, Department of Mechanical Engineering, McGill University, Montréal, \\ Québec, Canada H2A 2 A7 \\ H. KOMSUOḠLU \\ Artificial Intelligence Laboratory, University of Michigan, Ann Arbor, MI 48109, USA \\ M. BUEHLER \\ Ambulatory Robotics Laboratory, Department of Mechanical Engineering, McGill University, Montréal, \\ Québec, Canada H2A $2 A 7$ \\ H.B. BROWN JR. \\ The Robotics Institute, Carnegie Mellon University, Pittsburgh, PA 15213, USA \\ D. MCMORDIE \\ Ambulatory Robotics Laboratory, Department of Mechanical Engineering, McGill University, Montréal, \\ Québec, Canada H2A 2 A7 \\ U. SARANLI \\ Artificial Intelligence Laboratory, University of Michigan, Ann Arbor, MI 48109, USA \\ R. FULL \\ Department of Integrative Biology, University of California at Berkeley, Berkeley, CA 94720, USA \\ D.E. KODITSCHEK \\ Artificial Intelligence Laboratory, University of Michigan, Ann Arbor, MI 48109, USA
}

\begin{abstract}
RHex is an untethered, compliant leg hexapod robot that travels at better than one body length per second over terrain few other robots can negotiate at all. Inspired by biomechanics insights into arthropod locomotion, RHex uses a clock excited alternating tripod gait to walk and run in a highly maneuverable and robust manner. We present empirical data establishing that RHex exhibits a dynamical ("bouncing") gait—its mass center moves in a manner
\end{abstract}

\footnotetext{
*This work was supported in part by DARPA/SPAWAR under contract N66001-00-C-8026. Portions of the material reported here were first
} presented in a conference paper appearing in the collection (Altendorfer et al., 2000). 
well approximated by trajectories from a Spring Loaded Inverted Pendulum (SLIP)—characteristic of a large and diverse group of running animals, when its central clock, body mass, and leg stiffnesses are appropriately tuned. The SLIP template can function as a useful control guide in developing more complex autonomous locomotion behaviors such as registration via visual servoing, local exploration via visual odometry, obstacle avoidance, and, eventually, global mapping and localization.

Keywords: legged locomotion, hexapod robot, tripod gait, spring loaded inverted pendulum, (SLIP), hierarchical control, biomimesis, cockroach locomotion

\section{Introduction}

Animals inspire our intuition that legs may be necessary for satisfactory exploration of the highly broken and unstable landscapes prevailing on other planets. In this view, legged machines that capture some measure of animal mobility afford the best hope against the many inhospitable conditions extra-terrestrial settings impose. We have derived substantial inspiration from cockroach running in the design of a prototype robot, RHex, that breaks new ground in artificial legged locomotion (Saranli et al., 2000, 2001). Our breadbox sized, compliant leg hexapod, RHex (Fig. 1), travels at speeds better than one body length per second over terrain that few other robots can negotiate at all. The large and growing field of legged robotics includes a diverse array of laboratory vehicles inspired by arthropod locomotion, as well as at least one commercial crabinspired machine capable of sustained operation in the surf zone (iRobot, 1999). Yet, morphology notwithstanding, RHex arguably bears a closer relationship to Raibert's pioneering monopods (Raibert, 1986). For the hexapod literature has heretofore concerned static or quasi-static walking machines, while RHex can walk

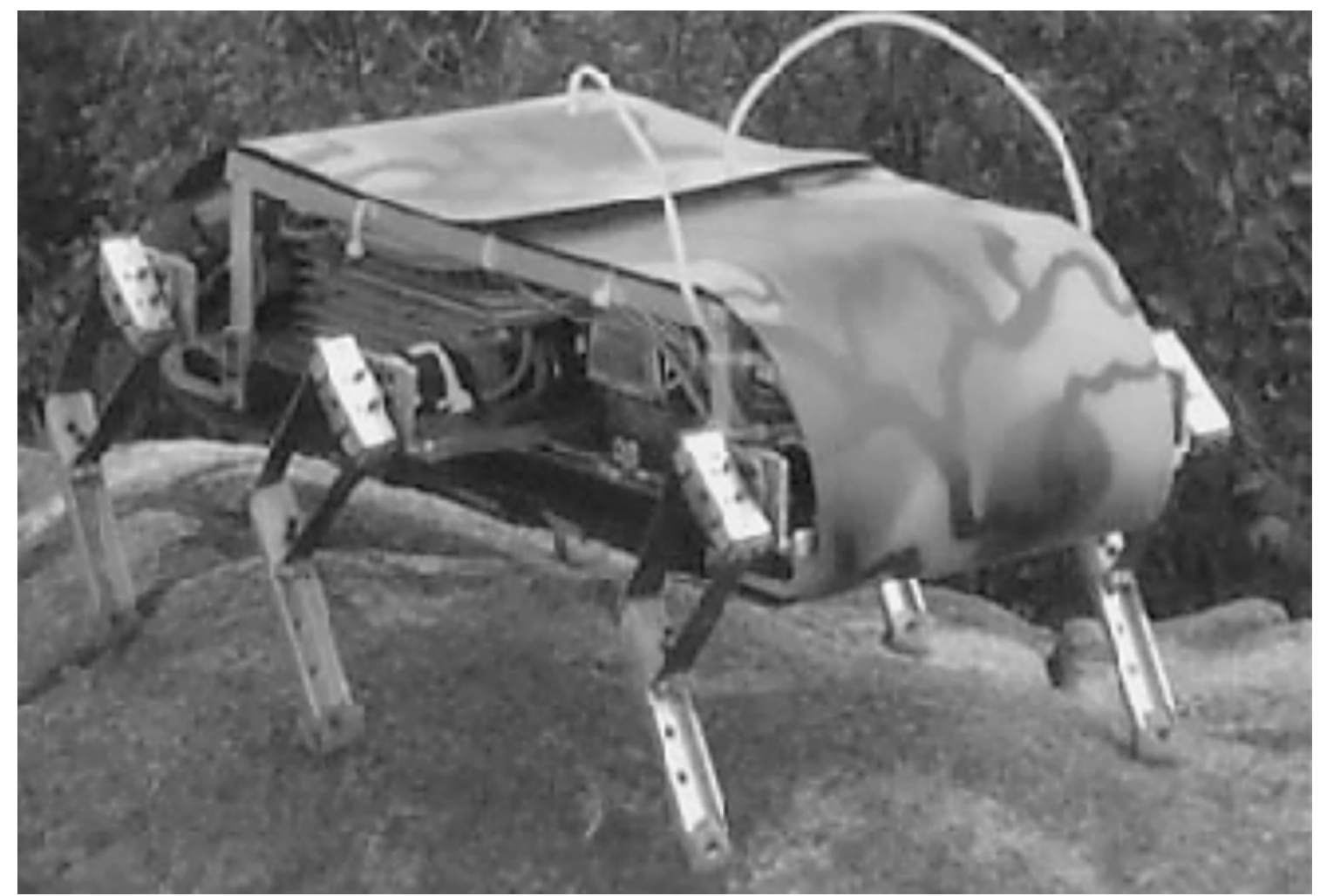

Figure 1. RHex on rock (www.RHex.net). 
and run. In this paper we present initial evidence establishing that RHex can "bounce" along its way as if it were indeed built like a pair of Raibert's pogo sticks, alternating in a $50 \%$ duty factor with no aerial phase. We will first review the biological inspiration motivating that observation, noting that cockroaches bounce along in the same manner, running, according to biomechanics, without ever leaving the ground. We end with somewhat more speculative remarks concerning its utility, imagining the benefits for stability and maneuverability.

\subsection{Biological Inspiration}

In considering the astonishing performance of the death-head cockroach, Blaberus discoidalis, over badly broken terrain, one of us (Full) has opined:

\begin{abstract}
"Simple feedforward motor output may be effective in negotiation of rough terrain when used in concert with a mechanical system that stabilizes passively. Dynamic stability and a conservative motor program may allow many-legged, sprawled posture animals to miss-step and collide with obstacles, but suffer little loss in performance. Rapid disturbance rejection may be an emergent property of the mechanical system." (Full et al., 1998)
\end{abstract}

While the materials and morphology displayed in Fig. 1 will not evoke in all viewers the image of a cockroach, their coordination in the generation of body motion is very strongly inspired by the following observations that underlie the previous quote.

Cockroach legs are (i) arranged in a sprawled posture affording (ii) significant overall compliance (Blickhan and Full, 1993) that may be (iii) excited by a strongly stereotypical "clock" reference signal (Kubow et al.) with the apparent (and surprising) consequence of a (iv) mechanically self-stabilized gait (Kubow and Full, 1999; Schmitt and Holmes, 2000). Thus, although RHex's body shape, leg design and materials are quite dissimilar from those of a cockroach, their coordination in the phasing of stance and swing are arranged so as to achieve a similar dynamical effect. The legs are attached in a (i) sprawled posture, spreading out so as to confer static stability at rest and low speed operation. Further, while each of RHex's 6 legs has only one actuated degree of freedom (at the hip), in contrast to the multiply actuated leg joints of a cockroach they incorporate (ii) a carefully tuned passive radial com- pliance. Finally, RHex exhibits a large suite of stable locomotion behaviors when each alternating tripod of hip motors is forced via a local proportional-derivative (PD) control law to track (iii) the identical (or, for the opposed tripod of legs, a $180^{\circ}$ out of phase) copy of a feedforward reference signal (Saranli et al., 2000). We presume (but cannot yet formally prove) that this feedforward stimulus excites a limit cycle (iv) in the coupled leg-body mechanism. We will present empirical evidence below that the projection of this putative limit cycle onto the mechanism's center of mass coordinates yields the motion of a specific pogo stick - the spring loaded inverted pendulum (SLIP) — when the controller and mechanical parameters are properly tuned.

Philosophically, RHex's design exemplifies our notion of biological inspiration in robotics, namely, that novel concept transfer from biology can increase performance in machines. This represents a cautionary approach that eschews "biology by default" (Ritzmann et al., 2000) or even morphologically identified design in favor of identification and mimicry of selected functional components. In the long run, we aim to articulate broad principles with mathematically precise formulations of biomechanical observed fact and then translate these into specific design practices. Meanwhile, pending that formal understanding, empiricism and intuition about biological function remains subservient to engineering practice in our work.

\subsection{Hierarchical Control}

Accumulating evidence in the biomechanics literature suggests that agile locomotion is organized in nature by recourse to a controlled bouncing gait wherein the "payload," the mass center, behaves mechanically as though it were riding on a SLIP (Blickhan and Full, 1993; Full and Farley, 2000). Given its ubiquity, we surmise that this specific form of dynamic locomotion must confer significant benefits upon animal runners. There is, indeed, clear evidence in some animal species that bouncing stores and returns energy (Biewener and Baudinette, 1995). For robots, storing periodically in a spring potential some portion of their fore-aft kinetic energy seems like an effective resolution to the inevitably constraining actuator torque and power limits. Limbs must move both quickly and forcefully in the high performance regimes we seek, but actuators cannot operate both at high speeds and at high torque levels. A careful exposition of the power and energy implications attendant upon a bouncing gait lies well 
beyond the scope of the present article. We explore instead the benefits of imposing this hierarchical organization on the control and stabilization architectures of complex, high degree of freedom mechanisms.

That exploration centers on the notion of the "template and anchor" hierarchy (Full and Koditschek, 1999). A template is a low dimensional model of a mechanism operating within a specified environment that is capable of expressing a specific task as the limit set of a suitably tuned dynamical system involving some controlled (robot) and un-controlled (environment) degrees of freedom. To "anchor" this low dimensional model in a more physically realistic higher degree of freedom representation of the robot and its environment, we seek controllers whose closed loops result in a low dimensional attracting invariant submanifold on which the restriction dynamics is a copy of the template.

Raibert's monopods were literal embodiments of the SLIP (Raibert, 1986). His bipeds functioned as alternating pogo sticks and his quadrupeds were constituted as virtual alternating pogo sticks. By recourse to such decompositions, he found it possible to impose relatively simple and largely decoupled stride-to-stride gait control laws over the within-stride regulation loops. One of us (Buehler), has pursued a more minimalist version of the Raibert quadrapeds in the form of passively compliant telescoping legs driven at each hip by only one actuator per leg in a design that might be seen as the immediate forebear of RHex (Buehler et al., 1998, 1999; Papadopoulos and Buehler, 2000).

Biomechanical evidence for the ubiquity of a SLIP template naturally suggests the possibility of pogo stick based control for more diverse leg morphologies. For example, a simulation study (Saranli et al., 1998) demonstrated an approximate anchoring of the SLIP template in a planar 4 DOF leg with ankle, knee and hip joints (AKH), similar in morphology to a human leg.

Raibert's machines were tethered to the wall by power cables. One central advance that RHex introduces is, of course, power autonomy. We believe that our insistence on one actuator only per leg-a remedy against the longstanding problem in robotics and automation of poor force and power density properties in commercially available actuators (Hollerbach et al., 1992)-represents the key enabler in this development. But the absence of any actuation beyond the torsional hip motors significantly complicates the decomposition methods outlined just above.
Following the insect example, we constitute RHex's gait from the alternation of two tripods that each act (relatively out of phase) as virtual monopod pogo sticks. However, in contrast to Raibert's mechanisms, none of RHex's legs has the literal morphology of the SLIP - they bend rather than telescope at the knee. Still more importantly, there is no direct affordance over the radial compliance in any of the physical limbs-all energy and phase manipulations throughout the machine must be introduced via hip torques.

In summary, RHex's essential difference from Raibert's machines rests in its greater number of legs placed in a sprawled tripod and in the absence of radially oriented actuators in the shanks. The virtue of the first change lies in the greater ease of traversal over broken terrain exploiting passive dynamic stability; vertical balance is far less of an issue (although pitching oscillations are still far from negligible and have the effect of destabilizing or at least badly perturbing the gait). The virtue of the second change lies in pulling out the wall plug. Can we, nevertheless, realize the supposed benefits of the SLIP template in this very different anchor?

\section{Finding SLIP in RHex's Motion}

In this section we discuss evidence suggesting the presence of certain "sweet spots" in the RHex parameter space, wherein the SLIP emerges naturally. It is still unclear whether this respects the formal "anchored template" paradigm wherein the lower dimensional dynamics actually appears as an attracting invariant dynamical submanifold. However, experimental evidence revealing the template behavior in steady state from various different initial conditions suggests there are, indeed, operating regimes where the system trajectories are attracted to the low dimensional SLIP template dynamics. Further evidence for the SLIP template comes from numerical studies (Saranli, 2000).

To determine whether RHex passively anchors a SLIP, the ground reaction forces produced by the robot during locomotion were measured over the course of 92 trials using two six-component force plates. ${ }^{1}$ The force and torque signals were amplified and each channel was recorded at $1000 \mathrm{~Hz}$ by an analog to digital converter. Each trial was also recorded by a high speed video camera. As discussed in detail in Altendorfer et al. (2000), of the two different leg designs tested, only the passive four bar linkage displayed in Fig. 1, had sufficiently low stiffness to permit operating in the 


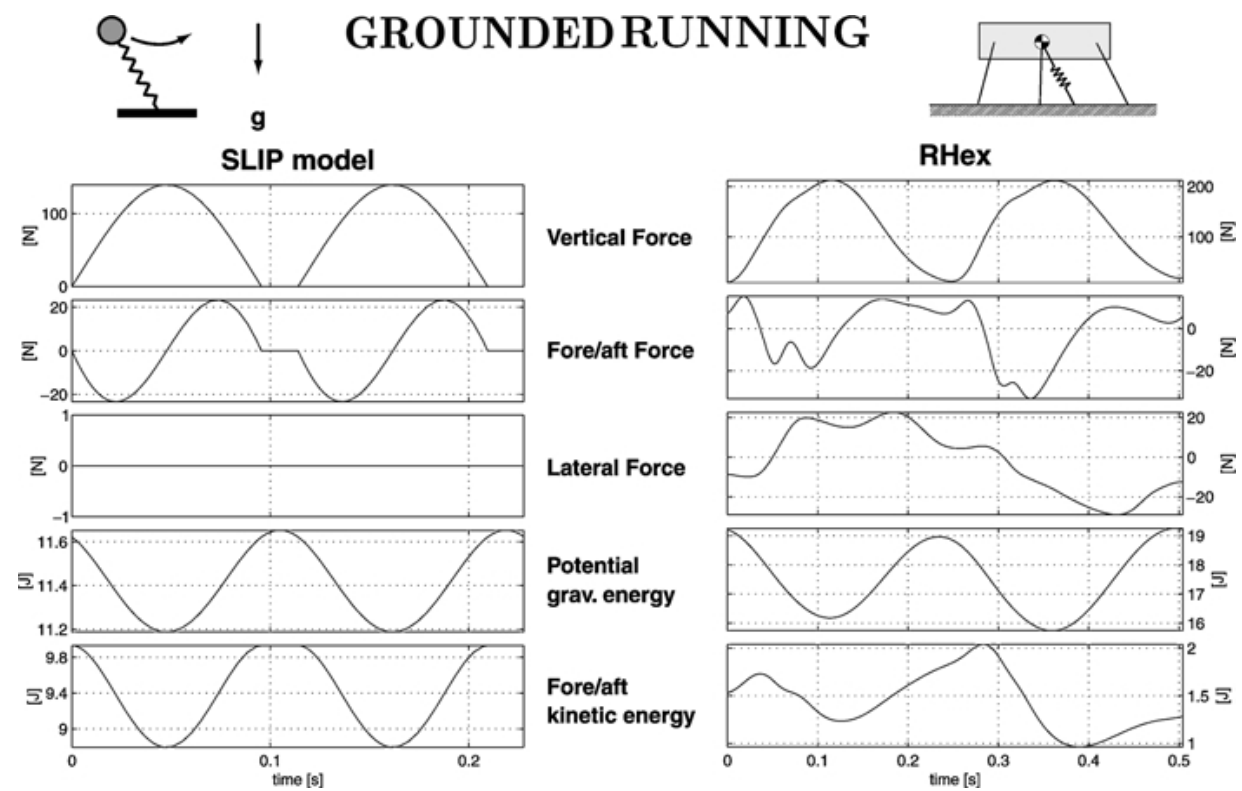

Figure 2. Comparison of sample RHex single stride data (no aerial phase) with SLIP predictions (aerial phase interval $\sim 5 \%$ of full cycle).

SLIP regime. Moreover, in order to further reduce the natural frequency of the effective vertical spring-mass system defined by the body-legs (down to the maximal stride frequency permitted by the limited motor power) we found it necessary to increase the payload mass incrementally from $7.83 \mathrm{~kg}$ to $9.47 \mathrm{~kg}$ to $11.12 \mathrm{~kg}$ to $11.94 \mathrm{~kg}$. In this highest mass regime we observed a transition from stiff virtual inverted pendulum (IP) to the desired SLIP reported below. There were 14 trials in this collection.

First, in order to help visualize what the fitting study establishes, we qualitatively compare force and energy data of a SLIP trajectory with short aerial phase and sample RHex data (Fig. 2). In particular, all time trajectories approximately enjoy the same phase relationships. Indeed, comparing SLIP and RHex data in the first and fourth row, the vertical force peaks at mid-step when the COM is at its lowest height-a necessary and characteristic property of SLIP equilibrium gaits. Also, comparing the fourth and the fifth row, gravitational potential energy and fore/aft kinetic energy of the COM fluctuate in phase. This "inphase" relationship between vertical height and horizontal kinetic energy also represents a key feature of SLIP locomotion, in contrast to inverted pendulum (IP) vaulting.

The SLIP template imposes a very particular set of relationships - those specified by the Lagrangian mechanics of a single point mass prismatic-revolute (i.e., polar coordinate) kinematic chain-between the ground reaction forces, motion of the COM, and system energies. Denoting by $b$ the position vector (relative to some inertial frame) in the saggital plane of the center of mass in cartesian coordinates, this relationship may be specified as $\ddot{b}=-D \phi(\|b\|) /\|b\|-g$, where $\phi$ is a spring potential normalized by mass and $g$ denotes the acceleration due to gravity. Ruina has pointed out (Ruina) that any convex curve supports in a neighborhood of its vertical minimum at least one time varying trajectory generated by some SLIP. RHex's saggital plane COM might well lie along a convex curve without establishing what we are interested in testing: whether its actual time trajectory along this curve can be readily generated by some SLIP model.

To this end, given a COM trajectory fragment with mass $m$, the COM position, and acceleration were used to fit via linear regression a Hooke spring law with unknown spring length $q_{r 0}$ and stiffness $\kappa$ (note this corresponds to setting $\phi(q)=(\kappa / 2 m)\left(q-q_{r 0}\right)^{2}$ in the equations of motion above). As an illustration of the fitting results, the best and worst SLIP fits are presented in Fig. 3. In all cases, we report rms error as a percentage of the rms value of the data trajectory (arithmetic mean of positions and velocities) itself. Thus, Fig. 3(a) shows that this worst trial yielded rms errors of $21.6 \%$, while 


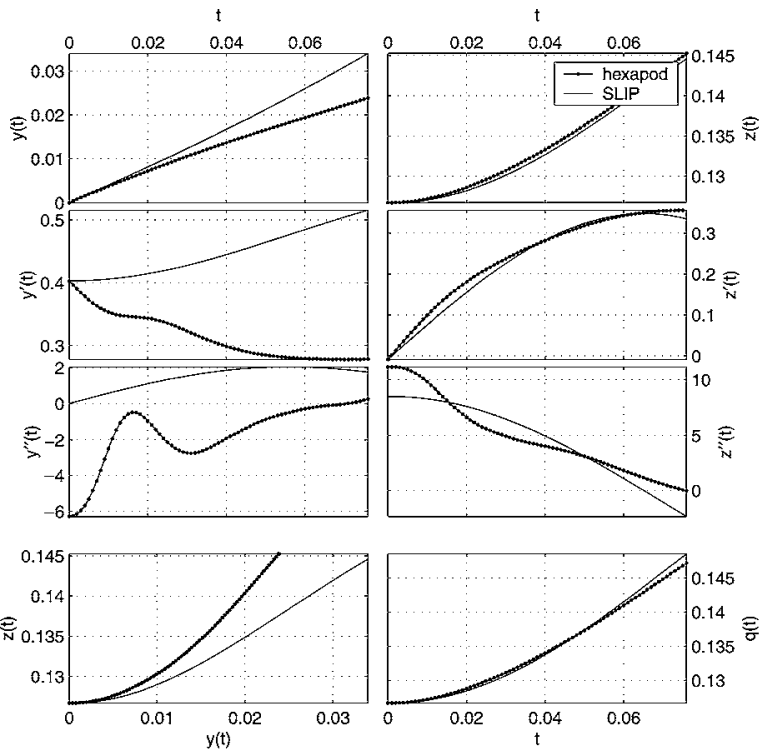

(a)
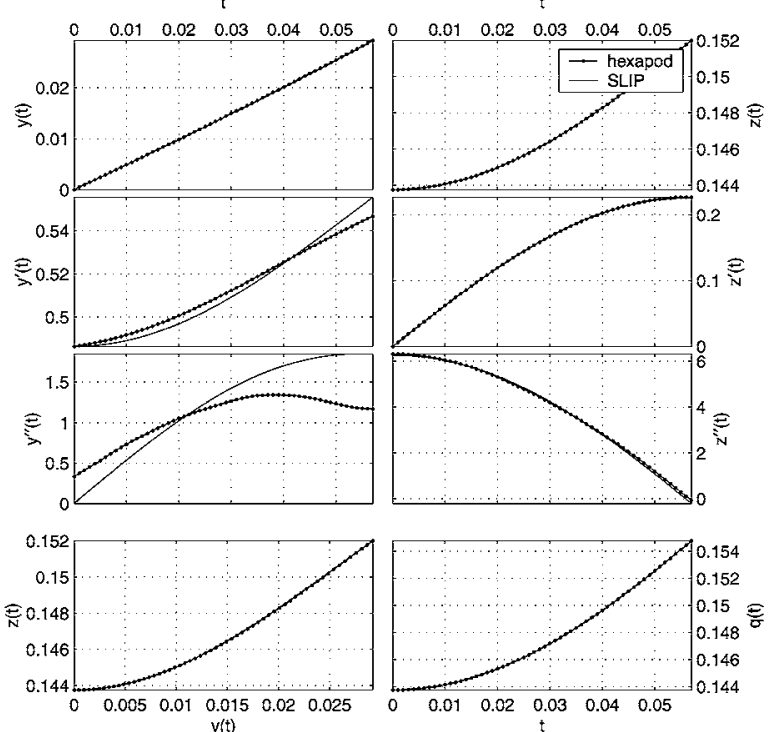

(b)

Figure 3. Worst and best SLIP fits: Dotted lines represent experimental data; solid lines represent fitted SLIP trajectories. This figure is taken from (Altendorfer et al., 2000). (a) Worst fitting trial with rms error $\Delta_{L_{2}}=21.6 \%$. (b) Best fitting trial with rms error $\Delta_{L_{2}}=0.3 \%$.

Fig. 3(b) shows that the best trial yielded an rms error of less than $1 \%$. On average, the ensemble of 14 trials generated a mean rms error of $7 \%$, with mean spring constant of $\kappa=6100 \mathrm{~N} / \mathrm{m}$. To get a bigger data sample, we ran a large number of simulations in SimSect in the same parameter range and obtained very similar results. We conclude that the SLIP dynamical system describes these data quite well.

\section{Conclusions}

Hierarchy promotes the use of few parameters to control complex systems with many degrees of freedom. In this light, as we understand matters, the emergence of a passively anchored SLIP in RHex is most fortunate. The pogo-stick can function as a useful control guide in developing more complex autonomous locomotion behaviors such as registration via visual servoing, local exploration via visual odometry, obstacle avoidance, and, eventually, global mapping and localization. In the longer term, we propose to work with the anchored SLIP in RHex in analogy to the manner in which the simple "Raibert vertical" template has been exploited in prior work by some of the authors and colleagues (Buehler et al., 1990) with simpler dynamically dexterous robots. Namely, as we shape behavior via manipulation of gains-in-the-loop (Burridge et al., 1999), we hope to develop a formal programming language with semantics in the world of dynamical attractors (Klavins and Koditschek, 2000).

Examples of the virtues of such decompositions and hierarchies at work in functioning robots include a series of batting machines that anchored the "Raibert vertical" template (Koditschek and Buehler, 1991) in a one degree of freedom paddle robot (operating into a two degree of freedom environment) (Buehler et al., 1990) and a three degree of freedom paddle robot (operating into a three degree of freedom environment) (Rizzi et al., 1992). This same idea is used to control a recently reported brachiating robot that anchors a rigid pendulum (Nakanishi et al., 2000). All of these robots were conceived as laboratory demonstration machines. In contrast, RHex's intrinsic robustness, stability and maneuverability suggest that there might be significant impact on applications if we succeed in transferring these ideas into the present setting.

Even now, prior to the imposition of more sophisticated hierarchical controllers, RHex's intrinsic characteristics-simple, rugged, light, autonomous, down-sizeable, reliable quasi-static operation, demonstrated dynamical capability (e.g., great jumping potential in reduced gravity environments) - all make RHex an ideal candidate for a planetary explorer. 


\section{Acknowledgments}

This work is supported by DARPA/ONR under contract N00014-98-1-0747 and DARPA/ SPAWAR under contract N66001-00-C-8026. We thank Rodger Kram and Claire Farley for the use of the force platform, Irv Scher for collaboration at an early stage of this project and William Schwind for the insight and the analytical tools he provided.

\section{Note}

1. Biomechanics Force Platform, Advanced Mechanical Technology, Inc., Newton, MA.

\section{References}

Altendorfer, R., Saranli, U., Komsuoḡlu, H., Koditschek, D.E., Brown Jr., H.B., Buehler, M., Moore, N., McMordie, D., and Full, R. 2000. Evidence for spring loaded inverted pendulum running in a hexapod robot. In Proceedings of the International Symposium on Experimental Robotics, Honolulu, HI.

Biewener, A.A. and Baudinette, R.V. 1995. In vivo muscle force and elastic energy storage during steady-speed hopping of tammar wallabies (Macropus eugenii). J. Exp. Biol., V198(N9):1829_ 1841.

Blickhan, R. and Full, R. 1993. Similarity in multilegged locomotion: Bouncing like a monopode. J. Comp. Physiol., A 173:509517.

Buehler, M., Battagila, R., Cocosco, A., Hawker, G., Sarkis, J., and Yamazaki, K. 1998. SCOUT: A simple quadraped that walks, climbs and runs. In Proceedings of the IEEE International Conference on Robotics and Automation, Leuven, Belgium, pp.17071712.

Buehler, M., Cocosco, A., Yamazaki, K., and Battagila, R. 1999. Stable open loop walking in quadruped robots with stick legs. In Proceedings of the IEEE International Conference on Robotics and Automation, Detroit, MI.

Buehler, M., Koditschek, D.E., and Kindlmann, P.J. 1990. A family of robot control strategies for intermittent dynamical environments. IEEE Control Systems Magazine, 10(2):16-22.

Burridge, R.R., Rizzi, A.A., and Koditschek, D.E. 1999. Sequential composition of dynamically dexterous robot behaviors. Int. J. Rob. Res., 18(6):534-555.

Full, R.J., Autumn, K., Chung, J.I., and Ahn, A. 1998. Rapid negotiation of rough terrain by the death-head cockroach. American Zoologist, 38:81A.

Full, R.J. and Farley, C.T. 2000. Musculoskeletal dynamics in rhythmic systems: A comparative approach to legged locomotion, In Biomechanics \& Neural Control of Posture \& Movement, J.M.
Winters and P.E. Crago (Eds.), Springer Verlag: New York, pp. 192-205.

Full, R.J. and Koditschek, D.E. 1999. Templates and anchors: Neuromechanical hypotheses of legged locomotion on land. J. Exp. Bio., 202:3325-3332.

Hollerbach, J.M., Hunter, I.W., and Ballantyne, J. 1992. A comparative analysis of actuator technologies for robotics. In The Robotics Review 2, O. Khatib, J.J. Craig, and T. Lozano-Perez (Eds.), MIT Press: Cambridge, Mass., pp. 299-342.

iRobot. 1999. Autonomous legged underwater vehicle (ALUV), http://www.irobot.com/rd/research_ariel.asp.

Koditschek, D.E. and Buehler, M. 1991. Analysis of a simplified hopping robot. International Journal of Robotics Research, 10(6):587-605.

Kubow, T.M. and Full, R.J. 1999. The role of the mechanical system in control: A hypothesis of self-stabilization in hexapedal runners. Phil. Trans. R. Soc. Lond., B 354:849-862.

Kubow, T., Garcia, M., Schwind, W., Full, R., and Koditschek, D.E. In preparation. A principal components analysis of cockroach steady state leg motion.

Klavins, E. and Koditschek, D.E. 2000. A formalism for the composition of concurrent robot behaviors. In Proc. IEEE Conf. Rob. and Aut., Vol. 4, pp. 3395-3402.

Nakanishi, J., Fukuda, T., and Koditschek, D.E. 2000. A brachiating robot controller. IEEE Trans. Rob. Aut., 16(2):109_ 123.

Papadopoulos, D. and Buehler, M. 2000. Stable running in a quadruped robot with compliant legs. In Proceedings of the IEEE International Conference on Robotics and Automation, San Francisco, CA.

Raibert, M.H. 1986. Legged Robots that Balance, MIT Press: Cambridge, MA.

Ruina, A. Personal communication.

Ritzmann, R.E., Quinn, R.D., Watson, J.T., and Zill, S.N. 2000. Insect walking and biorobotics: A relationship with mutual benefits. BioScience, 50(1):23-33.

Rizzi, A.A., Whitcomb, L.L., and Koditschek, D.E. 1992. Distributed real-time control of a spatial robot juggler. IEEE Computer, 25(5):12-24.

Saranli, U. 2000. SimSect hybrid dynamical simulation environment. University of Michigan Technical Report CSE-TR-437-00.

Saranli, U., Buehler, M., and Koditschek, D.E. 2000. Design, modeling and control of a compliant hexapod robot. In Proc. IEEE Int. Conf. Rob. Aut., San Francisco, CA, pp. 2589-2596.

Saranli, U., Buehler, M., and Koditschek, D.E. 2001. RHex-A simple and highly mobile hexapod robot. International Journal of Robotics Research (accepted).

Schmitt, J. and Holmes, P. 2000. Mechanical models for insect locomotion: Dynamics and stability in the horizontal plane I: Theory; II: Application. Biological Cybernetics, 83(6):501-515 and 517527.

Saranli, U., Schwind, W.J., and Koditschek, D.E. 1998. Toward the control of multi-jointed, monoped runner. In Proc. IEEE Int. Conf. on Rob. and Aut., Leuven, Belgium, pp. 2676-2682. 